Nig. J. Pure \& Appl. Sci. Vol. 34 (Issue 1, 2021)
e-ISSN 2756-4045
Life Sciences, Univ. of Ilorin, Nigeria
www.njpas.com.ng

\title{
http://dx.doi.org/10.48198/NIPAS/20.B19 Determination of Dependence of Attenuation on Material Thickness of Low-Density Polyethylene at Microwave Frequency
}

${ }^{1}$ Department of Physics, Federal University of Kashere, P. M. B. 0182. Gombe

${ }^{2}$ Department of Physics, University Putra Malaysia, Serdang 43400, Selangor, Malaysia

${ }^{3}$ Department of Physics, Gombe State University, P. M. B. 127, Gombe

${ }^{4}$ Department of Physics, Kaduna State University, P. M. B. 2339, Kaduna

\begin{abstract}
The attenuation of the electromagnetic wave signal due to power absorption by materials contributes to assessing the suitability of materials for use in microwave applications. One of the factors affecting power attenuation is material thickness. Therefore, this study investigates the effect of material thickness on the S-parameters and attenuation properties of low-density polyethylene (LDPE) at the X-band frequency range $(8 \mathrm{GHz}-12 \mathrm{GHz})$ to optimise the experimental design and reduce the cost associated with material preparation. The Waveguide measurement technique along with the finite element method (FEM) was implemented in COMSOL Multiphysics software for the study and analysis. The length of the waveguide was $200 \mathrm{~mm}$ while the length and width of the waveguide ports were $22.28 \mathrm{~mm}$ and $0.1143 \mathrm{~mm}$, respectively. The thickness of the LDPE was varied from $6 \mathrm{~mm}-14 \mathrm{~mm}$ with $2 \mathrm{~mm}$ increment. Variation of $S_{11}$ and $S_{21}$ as functions of material thickness were determined and analysed. $S_{11}$ decreased from 0.51 to 0.32 and $S_{21}$ increased from 0.85 to 0.93 at $6 \mathrm{~mm}$ and $14 \mathrm{~mm}$ of the material thickness, respectively. The attenuation of the LDPE due to power loss decreased from 1.37 to 0.31 at $6 \mathrm{~mm}$ and $14 \mathrm{~mm}$, respectively. It is determined from the results obtained that attenuation decreases as the thickness of the material increases, thereby reducing power loss due to absorption by the material.
\end{abstract}

Keywords: LDPE, S-parameters, Attenuation, Sample thickness

\section{Introduction}

Insight into dielectric materials response to an electromagnetic field (EM, Filed) at microwave frequency is vital especially for specialties such as electronics and communications, be it military, industry, or consumer applications ( Ahmad et al., 2016). A dielectric material is a material capable of storage and dissipation of energy when placed in an electromagnetic field. Such materials are employed for different applications at microwave frequency such as absorbers or printed circuit boards (PCBs). When a dielectric material is to serve as an absorber for Electromagnetic Interference Shielding (EMI Shielding), it is required to absorb almost all the incident EM waves on it (Khamis et al., 2020) whereas for PCB application it is to absorb ideally zero per cent of

Corresponding Author: Alhaji Ibrahim Abubakar

Department of Physics, Federal University of Kashere, P. M. B. 0182. Gombe Email: alhaji259@gmail.com 
the incident EM waves. In other words, for PCB application at microwave frequency, it is required that the dielectric material transmit almost all the incident EM waves upon it.

Page | 3893 Material attenuation characteristics depend on several factors such as frequency, dielectric properties, material thickness (Yakubu et al, 2014; Ahamd et al., 2016) and so on. In this work, we intend to study the dependence of attenuation on material thickness of Low-Density Polyethylene (LDPE) in-silico at X-band frequency using COMSOL software. This will help us to design our experiment efficiently by reducing the cost and time consumption therein. The X-band is chosen for its importance in Military communication, satellite, radar, and weather applications. This is due to its advantage of extremely low atmospheric

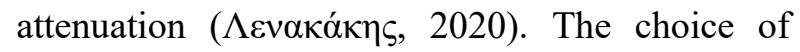
LDPE was based on its excellent dielectric properties, ease of processing due to its low melting point, and mechanical flexibility that makes it suitable for use in wearable electronics.

To calculate the attenuation of a sample, the transmission and reflection coefficients or Sparameters must be obtained through either analytical, experimental, or simulation method, therefore a good grasp on how to extract these parameters for the determination of attenuation capability of microwave materials.

COMSOL software is a 3-dimensional electromagnetic wave solution environment based on the finite element method (FEM) (Zimmerman, 2006). The results acquired from COMSOL for Sparameters agree with experimental measurements and so reliable that cumbersome theoretical analysis maybe jettisons in its favour (Yakubu et al, 2014; Khamis et al., 2020). There are several measurement techniques available for implementation into COMSOL to calculate the Sparameters but we prefer the waveguide technique for its design simplicity and accuracy (Abbas et al., 2001).

\subsection{Theory}

The finite element method (FEM) consists essentially of four (4) steps: (a) discretizing the region of solution into a finite number of elements, (b) deriving the governing equation for a given region, (c) gathering all the elements in a given region, and (d) Solving the systems of equations derived (Sadiku, 2018).

When a region of the solution is partitioned, the potential in the region is approximated as (Sadiku, 2018)

$V(x, y)=\sum_{e=1}^{N} V_{e}(x, y)$

Where $\mathrm{N}$ is the number of elements in the region. Thus, $V_{e}$ can be further written in a polynomial approximation as

$V_{e}(x, y)=a+b x+c y$

Therefore, the electric field in the region is thus given as

$E_{e}=-\nabla V_{e}=-\left(b \boldsymbol{a}_{x}+c \boldsymbol{a}_{\boldsymbol{y}}\right)$

When we consider a triangular element, fig. $1, V_{e}$ can be presented as

$V_{e}=\sum_{e=1}^{N} \alpha_{i}(x, y) V_{e i}$

Equation (4) is given as the potential at any point within the element when the potential at vertices are determined. The $\alpha_{i}$ is called the element shape and is given by (Sadiku, 2018)

$$
\begin{aligned}
& \alpha_{1}=\frac{1}{2 A}\left[\left(x_{2} y_{3}-x_{3} y_{2}\right)+\left(y_{2}-y_{3}\right) x+\right. \\
& \left.\left(x_{3}-x_{2}\right) y\right] \\
& \alpha_{2}=\frac{1}{2 A}\left[\left(x_{3} y_{1}-x_{1} y_{3}\right)+\left(y_{3}-y_{1}\right) x+\right. \\
& \left.\left(x_{1}-x_{3}\right) y\right] \\
& \alpha_{3}=\frac{1}{2 A}\left[\left(x_{1} y_{2}-x_{2} y_{1}\right)+\left(y_{1}-y_{2}\right) x+\right. \\
& \left.\left(x_{2}-x_{1}\right) y\right]
\end{aligned}
$$

And $A$ is the area of the elements in the region it is calculated as 
$A=\frac{1}{2}\left[\left(x_{2}-x_{1}\right)\left(y_{3}-y_{1}\right)-\left(x_{3}-x_{1}\right)\left(y_{2-} y_{1}\right)\right.$

The energy associated with all the elements assembled in the solution region is (Sadiku, 2018)

Page | 3894

$W=\sum_{e=1}^{N} W_{e}=\frac{1}{2} \epsilon[V]^{T}[C][V]$

Where $[V]$ is the potential, $[C]$ is the general coefficient matrix, and $\mathrm{N}$ is the number of the elements in the given region (Jin, 2014; Sadiku, 2018).

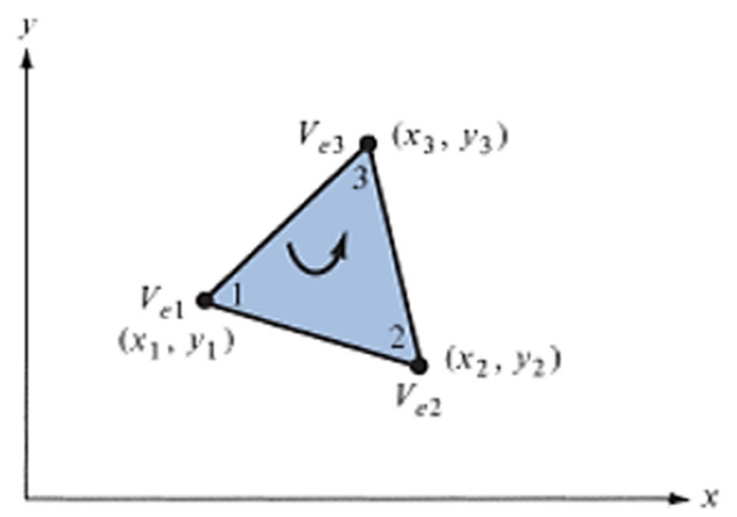

Fig. 1: Local triangular element

For the S-parameters, let's consider figure 2. Where the wave propagation in a transmission line filled with a dielectric sample is depicted. The electric field intensity in these three regions are denoted as $E_{I}, E_{I I}$, a and $E_{I I I}$ respectively (Soleimani et al., 2012; Wang and Hashiguchi, 2019).

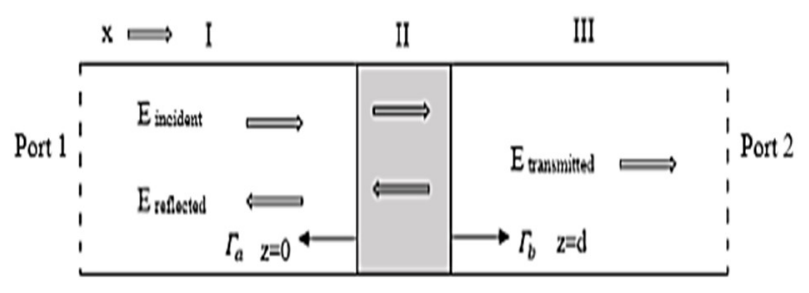

Fig. 2: Incident, Reflected, and Transmitted Electromagnetic Waves

Considering medium II, the magnitude of the Sparameters can be obtained by invoking the boundary conditions at $z=0$ and $z=d$. Thus,
$S_{11}=\frac{\Gamma_{a}+\Gamma_{b} P_{\text {theory }}^{2}}{1+\Gamma_{a} \Gamma_{b} P_{\text {theory }}^{2}}$

$S_{21}=\frac{1+\Gamma_{a} \Gamma_{b} P_{\text {theory }}}{1+\Gamma_{a} \Gamma_{b} P_{\text {theory }}^{2}}$

Where

$$
\begin{gathered}
\Gamma_{a}=\frac{\left(Z_{s}-Z_{i}\right)}{\left(Z_{s}+Z_{i}\right)}=\frac{\left(\sqrt{\varepsilon_{\mathrm{I}}^{*}}-\sqrt{\left.\varepsilon_{\mathrm{S}}^{*}\right)}\right.}{\left(\sqrt{\varepsilon_{\mathrm{I}}^{*}}+\sqrt{\left.\varepsilon_{\mathrm{S}}^{*}\right)}\right.} \\
\Gamma_{b}=\frac{\left(Z_{I I I}-Z_{S}\right)}{\left(Z_{I I I}+Z_{S}\right)}=\frac{\left(\sqrt{\varepsilon_{\mathrm{S}}^{*}}-\sqrt{\varepsilon_{\mathrm{III}}^{*}}\right)}{\left(\sqrt{\varepsilon_{\mathrm{S}}^{*}}+\sqrt{\varepsilon_{\mathrm{III}}^{*}}\right)} \\
P_{\text {theory }}=e^{\left(-\gamma_{S} d\right)}
\end{gathered}
$$

The $\Gamma_{a}$ and $\Gamma_{b}$ describes the magnitude of reflection coefficients at the boundaries $z=0$ and $z=d$ while $P_{\text {theory }}$ indicates the propagation factor due to the dielectric sample at medium II. Assuming medium I and III are identical then $\Gamma_{a}=-\Gamma_{b}$. Therefore equations (7) and (8) can be re-written as (Baker-Jarvis et al, 1990 and Soleimani et al., 2012)

$S_{11}=\frac{\left(1-P_{\text {theory }}^{2}\right) \Gamma_{a}}{1-P_{\text {theory }}^{2} \Gamma_{a}^{2}}$

$S_{21}=\frac{\left(1-P_{\text {theory }}\right) \Gamma_{a}^{2}}{1-P_{\text {theory }}^{2} \Gamma_{a}^{2}}$

It is to be noted the scattering matrix is symmetrical i.e. $S_{12}=S_{21}$.

The attenuation is obtained from equation 11 (AF et al., 2016; Yakubu et al., 2015).

attenu ation $=-20 \log _{10}\left(S_{21}\right)$

\subsection{Simulation}

There are five steps to be taken before any simulation session is run in the COMSOL software. First, a 3-dimensional plane is chosen and the RF module for electromagnetic waves in the frequency domain is selected. Then the geometry of the structure to be modelled is constructed which is waveguide in this case. Next appropriate physical constraints and boundary 
conditions are then applied to the geometry. The meshing of the structure follows while the operating frequency is assigned before the session is finally run.

Page | 3895 The dimension of the waveguide being designed is $0.2 \times 0.1143 \times 0.0228 \mathrm{~m}$ while the thickness of the dielectric sample was varied from $6-14 \mathrm{~mm}$. The permittivity of the LDPE used was $2.2-j 0.0004$. This implies that the dielectric constant $=2.2$ and loss factor $=0.0004$.

\section{Results and Discussion}

Results of S-parameters variation due to frequency change at different thicknesses are presented in figure 3. The value for the transmission coefficient, $S_{21}$, is higher than the reflection coefficient, $S_{11}$, which is in agreement with the theory in section 2.0. Figure $3 \mathrm{a}$ and $3 \mathrm{~b}$ reveal that at 6 and $8 \mathrm{~mm}$ sample thickness, the value of $S_{21}$ is lowest and at $8 \mathrm{GHz}$ and highest at $12 \mathrm{GHz}$ inching towards unity while the value for $S_{11}$ is highest at $8 \mathrm{GHz}$ and lowest at $12 \mathrm{GHz}$ approaching zero. This shows that for a thin sample, $S_{21}$ and $S_{11}$ have direct and indirect proportionality with the frequency of operation and $\mathrm{X}$-band region.

For figure $3 \mathrm{c}, 3 \mathrm{~d}$ and $3 \mathrm{e}$ which corresponds with sample thicknesses of 10,12 , and $14 \mathrm{~mm}$, the values of $S_{11}$ and $S_{21}$ were the lowest and highest at 11 , 9.5 and $8.5 \mathrm{GHz}$ respectively. This implies that for material thickness $10 \mathrm{~mm}$, the resonant frequency is found at $11 \mathrm{GHz}$ where the maximum power is transmitted through the LDPE dielectric material. The same argument goes for thickness of 12 and $14 \mathrm{~mm}$ with their resonant frequency obtained at 9.5 and $8.5 \mathrm{GHz}$ respectively.

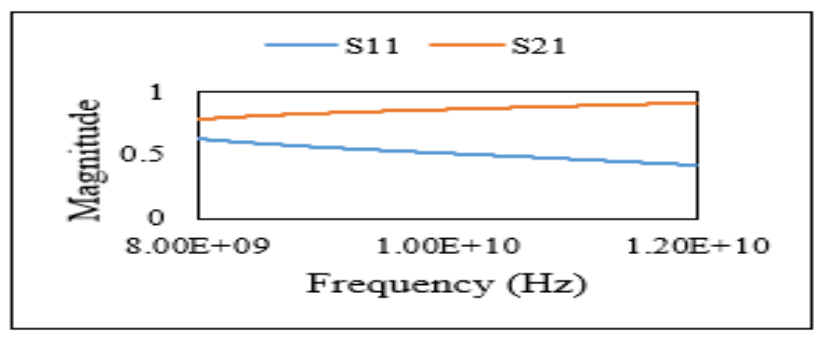

(a)

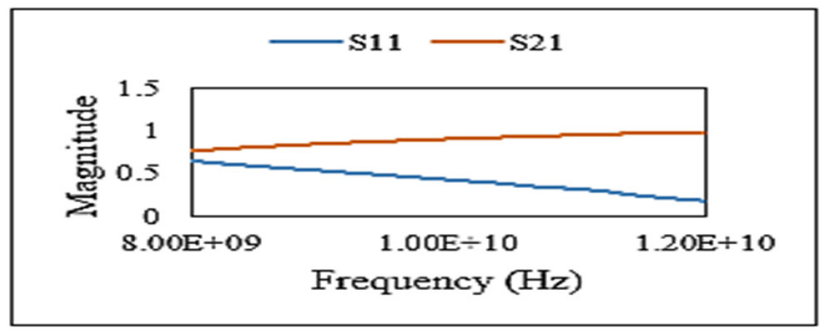

(b)

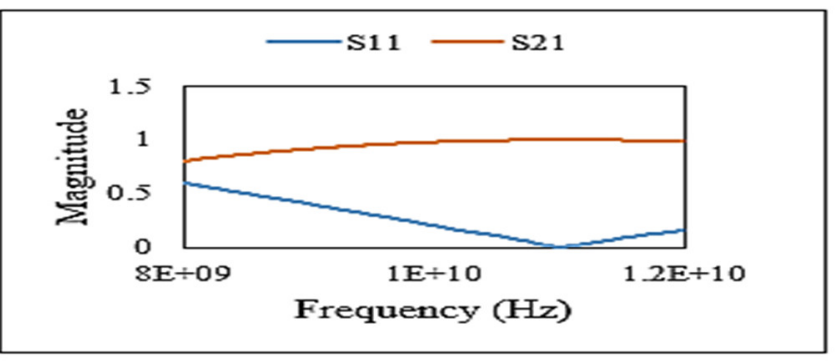

(c)

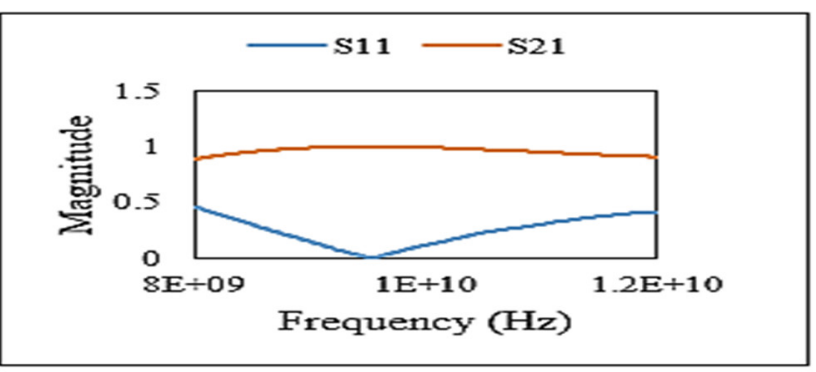

(d)

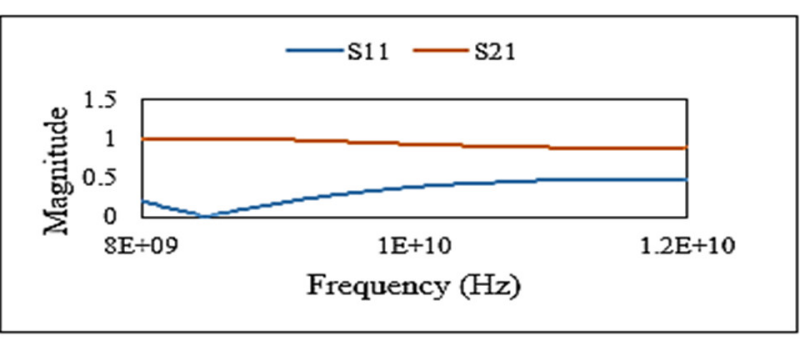

(e)

Fig. 3: The S-parameters for 6, 8, 10, 12, and 14mm thicknesses respectively 
The attenuation of different LDPE material thickness in the $\mathrm{x}$-band frequency is presented in figure 4 below. It is clearly shown in the figure that the highest values for attenuation for 6 and $8 \mathrm{~mm}$ thicknesses were obtained at $8 \mathrm{GHz}$ while the lowest

Page | 3896 values of the attenuation at $12 \mathrm{GHz}$ even though their resonant frequencies were not found.

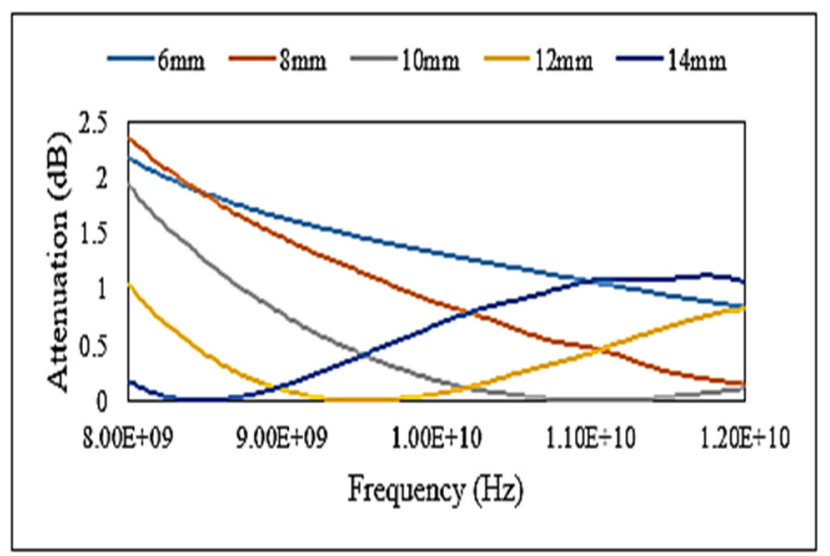

Fig. 4: Attenuation (dB) for different thicknesses at $\mathrm{x}$-band frequency

For 10,12 , and $14 \mathrm{~mm}$, the attenuation levels were 2,1 , and $0.15 \mathrm{~dB}$ at $8 \mathrm{GHz}$ before dropping to almost zeros at $11,9.5$ and $8.5 \mathrm{GHz}$ respectively. This shows that at these frequencies the power is maximally transferred and utilizing LDPE material with the respective thickness at these resonant frequencies will be suitable for PCB applications such as substrates for microstrip antennas.

Table 1: Mean values of S-parameter with the corresponding Attenuation at different thicknesses

\begin{tabular}{lrrl}
\hline $\begin{array}{l}\text { Thickness } \\
(\mathrm{mm})\end{array}$ & $S_{11}$ & $S_{21}$ & Attenuation(dB) \\
\hline 6 & 0.51485 & 0.854453 & 1.373691 \\
8 & 0.42294 & 0.894032 & 0.994821 \\
10 & 0.248704 & 0.949822 & 0.463755 \\
12 & 0.241988 & 0.961006 & 0.350165 \\
14 & 0.320908 & 0.933224 & 0.610521 \\
\hline
\end{tabular}

Table 1 gives the mean values for S-parameters and attenuation for different thicknesses at $\mathrm{x}$-band frequency which implies that mean attenuation increases with an increase in material thickness with the only exception at $14 \mathrm{~mm}$ thickness. This is because the resonant frequency at $14 \mathrm{~mm}$ was found at $8.5 \mathrm{GHz}$ slightly above $8 \mathrm{GHz}$, the origin of the frequency band.

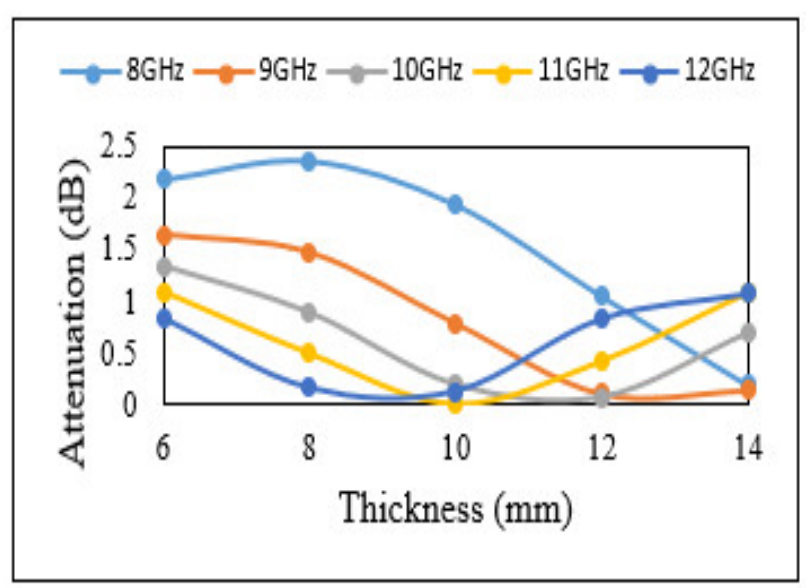

Fig. 5: Attenuation (dB) for different thicknesses at $8,9,10,12 \mathrm{GHz}$ respectively

Figure 5 presents attenuation levels for different frequencies in the band for $6-14 \mathrm{~mm}$ thickness. It is indicated that the attenuation values at $8 \mathrm{GHz}$ were lowest and highest at 8 and $14 \mathrm{~mm}$ thickness respectively. This implies that maximum power is transferred at $8 \mathrm{GHz}$ for $14 \mathrm{~mm}$ thickness. Similarly, for operation at $9 \mathrm{GHz}$ utilizing LDPE material for maximum power transfer will be recommended at $12 \mathrm{GHz}$ whereas for 10,11 , and $12 \mathrm{GHz}, \mathrm{LDPE}$ of material thicknesses 12,10 , and $10 \mathrm{~mm}$ are recommended respectively.

\section{Conclusion}

The study of attenuation properties of different LDPE thicknesses was carried out using FEM through COMSOL software version 3.5. The Sparameters were obtained for the material thickness varied from $6-14 \mathrm{~mm}$ at $\mathrm{x}$-band frequency, i.e., 812GHz. The mean $S_{11}, S_{21}$ and attenuation for different thicknesses were found to be $0.51,0.85$, and 1.37 for $6 \mathrm{~mm}, 0.42,0.89$, and 0.99 for $8 \mathrm{~mm}$, $0.25,0.95$, and 0.46 for $10 \mathrm{~mm}, 0.24,0.94$, and 0.35 
for $12 \mathrm{~mm}$ and finally $0.32,0.93$, and 0.61 for $14 \mathrm{~mm}$ respectively. Further analysis shows that although the resonant frequencies were not found for 6 and $8 \mathrm{~mm}$, they have the lowest values of attenuation at $12 \mathrm{GHz}$. The resonant frequencies for 10,12 , and

Page | $389714 \mathrm{~mm}$ thicknesses were found at 11,9.5 and $10 \mathrm{GHz}$ respectively. The attenuation obtained for different LDPE thicknesses keeps decreasing until resonant frequency for any given thickness is found. This demonstrates the dependence of attenuation on material thickness.

\section{Conflict of interest}

Authors declare no conflict of interest whatsoever.

\section{References}

Abbas, Z., Pollard, R. D., and Kelsall, R. W. (2001). Complex Permittivity Measurements at Ka-Band Using Rectangular Dielectric Waveguide. Ieee Transactions on Instrumentation and Measurement, 50(5), 1334-1342.

Ahmad, A. F, A., Abbas, Z, ., Obaiys S. J, and Abdalhadi D. M. (2016). Attenuation Performance of Polymer Composites Incorporating NZF Filler for Electromagnetic Interference Shielding at Microwave Frequencies. Journal of Material Science \& Engineering, 05(06), 6$11 . \quad$ https://doi.org/10.4172/21690022.1000289

Baker-Jarvis, J., Vanzura, E. J., and Kissick, W. A. (1990). Improved Technique for Determining Complex Permittivity with the Transmission / Reflection Method. IEEE Transactions on Microwtheory And Techniques, 38(8), 1096-1103.

Jin, J.-M. (2014). The Finite Element Method In Electromagnetics (Third Edit). Wiley.

Khamis, A. M., Abbas, Z., Ahmad, A. F., Azis, R. S., Abdalhadi, D. M., and Mensah, E. E. (2020). Experimental and computational study on epoxy resin reinforced with microsized OPEFB using rectangular waveguide and finite element method. IET Microwaves, Antennas and Propagation, 14(8), 752-758. https://doi.org/10.1049/ iet- map.2019.0085

Meli, A. D., Abbas, Z., Zaid, M. H. M., and Ibrahim, N. A. (2019). The effects of SLS on the structural and complex permittivity of SLS-HDPE composites. Advances in Polymer Technology, 2019. https://doi.org/10.1155/2019/3420925

Sadiku, M. N. O. (2018). Element of Electromagnetics (7th Edition). New York: Oxford University Press.

Soleimani, H., Abbas, Z., Yahya, N., and Soleimani, H. (2012). Determination of complex permittivity and permeability of lanthanum iron garnet filled PVDFpolymer composite using rectangular waveguide and Nicholson - Ross - Weir ( NRW) method at X-band frequencies. Measurement, 45(6), 1621-1625. https://doi.org/10.1016/j.measurement.201 2.02.014

Wang, J., and Hashiguchi, M. (2019). Numerical Evaluation on Cutoff Frequency of Twisted Waveguide with Rectangular Crosssection. 1(1), 2-4.

Yakubu, A., Abbas, Z., and Hashim, M. (2014). Effect of material thickness on attenuation (dB) of PTFE using finite element method at X-band frequency. Advances in Materials Science and Engineering, 2014(2), 10-15. https://doi.org/10.115 $5 / 2014 / 965912$

Yakubu, A., Abbas, Z., Ibrahim, N., and Fahad, A. (2015). The Effect of $\mathrm{ZnO}$ nanoparticles Filler on Complex Permittivity of ZnO-PCL Nanocomposite at Microwave Frequency. Physical Science International Journal, 
6(3), 196-202. https://doi.org/10.97 34/psij/2015/1 6692

Zimmerman, W. B. J. (2006). Multiphysics Modeling with Finite Element Methods (1st Page | 3898


Retrieved from https://www.everythi ngrf.com/community/x-band on $8^{\text {th }}$ September 2020. 